ELECTRONIC RESEARCH ANNOUNCEMENTS OF THE AMERICAN MATHEMATICAL SOCIETY

Volume 3, Pages 126-130 (December 17, 1997)

S $1079-6762(97) 00037-1$

\title{
INVARIANTS OF TWIST-WISE FLOW EQUIVALENCE
}

\author{
MICHAEL C. SULLIVAN \\ (Communicated by Jeff Xia)
}

\begin{abstract}
Twist-wise flow equivalence is a natural generalization of flow equivalence that takes account of twisting in the local stable manifold of the orbits of a flow. Here we announce the discovery of two new invariants in this category.
\end{abstract}

\section{Flow equivalence}

Square nonnegative integer matrices are used to describe maps on Cantor sets known as subshifts of finite type. Two such matrices are flow equivalent if their induced subshifts of finite type give rise to topologically equivalent suspension flows. Here topologically equivalent just means there is a homeomorphism, taking orbits to orbits, while preserving the flow direction. A matrix $A$ is irreducible if for each $(i, j)$ there is a power $n$ such that the $(i, j)$ entry of $A^{n}$ is nonzero. In terms of the corresponding subshift and suspension, irreducibility is equivalent to the existence of a dense orbit. Irreducible permutation matrices give rise to flows with a single closed orbit and are thus said to form the trivial flow equivalence class. For nontrivial irreducible incidence matrices John Franks has shown that flow equivalence is completely determined by two invariants, the Parry-Sullivan number and Bowen-Franks group. Let $A$ be an $n \times n$ incidence matrix. Then

$$
P S(A)=\operatorname{det}(I-A) \quad \text { and } \quad B F(A)=\frac{\mathbb{Z}^{n}}{(I-A) \mathbb{Z}^{n}}
$$

are the Parry-Sullivan number and the Bowen-Franks group respectively. See [8], [1], and [2] or the recent text [6]. Huang has settled the difficult classification problem arising when the assumption of irreducibility is dropped, [3], [4], [5].

\section{Twist-Wise FLOW EQUivalence}

Represent $\mathbb{Z}_{2}$ by $\{1, t\}$, under multiplication with $t^{2}=1$. Let $A(t)$ be an $n \times n$ matrix with entries of the form $a+b t$, with $a$ and $b$ nonnegative integers. That is $A$ is a matrix over the semigroup ring $\mathbb{Z}^{+} \mathbb{Z}_{2}$. Call such a matrix a twist matrix. One interpretation of twist matrices is as follows. Suppose the suspension flow for $A(1)$ is realized as a 1-dimensional basic set, $\mathcal{B}$, of saddle type, of a flow on a 3-manifold. For each orbit in $\mathcal{B}$ there is a 2 -dimensional local stable manifold, a ribbon, if you like. Call the union of such ribbons the ribbon set, and denote it by $\mathcal{R}$. Each ribbon

Received by the editors June 18, 1997 and, in revised form, October 4, 1997.

1991 Mathematics Subject Classification. Primary 58F25, 58F13; Secondary 58F20, 58F03.

Key words and phrases. Dynamical systems, flows, subshifts of finite type.

(c)1997 American Mathematical Society 
is either an annulus, a Möbius band, or an infinity long strip. Now, $A(1)$ is the incidence matrix for the first return map $\rho$ on the rectangles of a Markov partition, $\left\{R_{1}, \ldots, R_{n}\right\}$, of a cross section of a neighborhood of $\mathcal{B}$. Thus, $A_{i j}(1)$ is the number of times $R_{i}$ passes through $R_{j}$. If we orient the rectangles, then we can let $a_{i j}$ be the number of components of $\rho\left(R_{i}\right) \cap R_{j}$ where orientation is preserved, and $b_{i j}$ be the number of components where orientation is reversed by the action of $\rho$. Then $A_{i j}(t)=a_{i j}+b_{i j} t$.

It is not necessary that the manifold be 3-dimensional or that there be only one stable eigenvalue. We only need a means of assigning orientations to rectangles of a Markov partition. We note that $A(-1)$ is related to the structure matrix of [1]. Two ribbon sets are topologically equivalent if there is a homeomorphism between them that preserves the flow direction. This leads us to define two twist matrices to be twist-wise flow equivalent if they induce topologically equivalent ribbon sets.

Theorem 2.1. The numbers $P S(A( \pm 1))$ and the groups $B F(A( \pm 1))$ are invariants of twist-wise flow equivalence.

It is clear that $P S(A(1))$ and $B F(A(1))$ are invariants in this category. In [9] it is shown that $P S(A(-1))$ is also invariant and it can now be reported that $B F(A(-1))$ is too [10]. We define an additional invariant in $\S 4$. However, we still do not possess a complete set of invariants. See $\S 6$.

\section{Matrix MOVes}

Twist-wise flow equivalence (or twist equivalence for short) is generated by three matrix moves [9]. These are called, the shift move, the expansion move, and the twist move, and are denoted by $\stackrel{s}{\sim}, \stackrel{e}{\sim}$ and $\stackrel{t}{\sim}$, respectively. The first two generate flow equivalence [8]. We define them below. Shift: $A \stackrel{s}{\sim} B$ if there exist rectangular matrices $R$ and $S$, over $\mathbb{Z}^{+} \mathbb{Z}_{2}$, such that $A=R S$ and $B=S R$. Expansion: $A \stackrel{e}{\sim} B$ if $A=\left[A_{i j}\right]$ and

$$
B=\left[\begin{array}{cccc}
0 & A_{11} & \cdots & A_{1 n} \\
1 & 0 & \cdots & 0 \\
0 & A_{21} & \cdots & A_{2 n} \\
\vdots & \vdots & & \vdots \\
0 & A_{n 1} & \cdots & A_{n n}
\end{array}\right]
$$

or vice versa. Twist: $A \stackrel{t}{\sim} B$ if $A=\left[A_{i j}\right]$ and

$$
B(t)=\left[\begin{array}{cccc}
A_{11} & t A_{12} & \cdots & t A_{1 n} \\
t A_{21} & A_{22} & \cdots & A_{2 n} \\
\vdots & \vdots & & \vdots \\
t A_{n 1} & A_{2 n} & \cdots & A_{n n}
\end{array}\right] .
$$

The shift move includes relabeling, so the expansion and twist moves can be done on other "locations" in the matrix. See [9] for geometric motivations.

\section{THE DOUble COVER FLOW}

We now consider another means of encoding the twisting of a ribbon set. For a 2 -dimensional ribbon set $\mathcal{R}$ place a flow on the boundary with direction parallel to the flow on its core $\mathcal{B}$. Call this the double cover flow of $\mathcal{B}$. An incidence matrix 
$D A$ can be constructed from a twist matrix $A(t)$ by replacing each entry $a+b t$ with $\left[\begin{array}{ll}a & b \\ b & a\end{array}\right]$. This amounts to using the matrix representation

$$
\mathbb{Z}_{2} \cong\left\{\left[\begin{array}{ll}
1 & 0 \\
0 & 1
\end{array}\right],\left[\begin{array}{ll}
0 & 1 \\
1 & 0
\end{array}\right]\right\}
$$

As this process is completely formal we need not be restricted to 2-dimensional ribbon sets. It is easy to show that $P S(D A)$ and $B F(D A)$ are invariants of twist equivalence. However, $P S(D A)=P S(A(+1)) \times P S(A(-1))$, and thus contains no new information. But $B F(D A)$ does distinguish twist matrices that the other invariants do not. Yet $P S(A( \pm 1)), B F(A( \pm 1))$, and $B F(D A)$ are not complete. See $\S 6$.

\section{Other Abelian groups}

If we replace $\mathbb{Z}_{2}$ with an arbitrary Abelian group $G$, the only major change is that the twist move must be replaced by a series of $g$-moves for each $g \in G$ defined by multiplying the first row by $g$ and the first column by $g^{-1}$. The Parry-Sullivan invariant becomes an element of the group ring $\mathbb{Z} G$. In [1] the Bowen-Franks group was defined for arbitrary Abelian groups, so there should be no trouble there. If one has a matrix representation of $G$, then even an analog of the double cover operation should go through. Suppose one has a finite directed graph and an associated subshift of finite type. Let the edges be labeled with elements of $G$ and suppose we seek conjugacies that preserve this additional information. Each closed orbit is paired with the product of its edge labels and this pairing is to be preserved under conjugacies. Then the analogs of the Parry-Sullivan and Bowen-Franks invariants should be useful. We are hopeful that researchers in other areas of dynamics or coding theory will find that they can exploit these ideas.

\section{EXAmples}

Consider $2 \times 2$ matrices with entries 0,1 , or $t$, but which are irreducible and nontrivial. For brevity we shall denote $\left[\begin{array}{ll}a & b \\ c & d\end{array}\right]$ by abcd. We will divide these matrices into 6 classes:

- $A=\{1111,1110,1101,1011,1 t t 1,1 t t 0,0 t t 1\}$,

- $B=\{t 111,111 t, t t t 1,1 t t t\}$,

- $C_{1}=\{1 t 11,11 t 1\}$,

- $C_{2}=\{t t 11, t 1 t 1,1 t 1 t, 11 t t, 1 t 10,11 t 0,0 t 11,01 t 1, t 110, t t t 0,011 t, 0 t t t\}$,

- $D=\{t 11 t, t t t t, t t 10, t 1 t 0,01 t t, 0 t 1 t\}$,

- $E=\{t 1 t t, t t 1 t\}$.

Within each of these classes we have shown that the matrices are twist equivalent by constructing the necessary matrix moves. In Table 1 we list the invariants for each class. Classes $A$ and $B$ cannot be distinguished, yet their ribbon sets cannot be homeomorphic since the ribbon set for $B$ contains Möbius bands whereas the closed ribbons for $A$ are all annuli. Hence our invariants are not complete. Of course, one can simply take orientability as an additional invariant. The frustrating point is that the motivation behind all the new invariants was precisely to capture orientation data. The classes $C_{1}$ and $C_{2}$ also have the same set of invariants. However, we have not been able to tell if they form a single twist class or not. 
TABLE 1

\begin{tabular}{|c|r|r|r|c|c|}
\hline Class & $P S^{+}$ & $B F^{+}$ & \multicolumn{1}{|c|}{$P S^{-}$} & $B F^{-}$ & $B F^{D}$ \\
\hline \hline$A$ & -1 & 0 & -1 & 0 & 0 \\
\hline$B$ & -1 & 0 & -1 & 0 & 0 \\
\hline$C_{1}$ & -1 & 0 & 1 & 0 & 0 \\
\hline$C_{2}$ & -1 & 0 & 1 & 0 & 0 \\
\hline$D$ & -1 & 0 & 3 & $\mathbb{Z}_{3}$ & $\mathbb{Z}_{3}$ \\
\hline$E$ & -1 & 0 & 5 & $\mathbb{Z}_{5}$ & $\mathbb{Z}_{5}$ \\
\hline
\end{tabular}

In Table 2 we have listed the invariants for a sampling of $3 \times 3$ matrices. Several features stand out. Any finitely generated Abelian group can be realized as a $B F(A(1))$ group [2], and we think this is likely true for $B F(A(-1))$ as well. However, certain groups do not seem to show up as Bowen-Franks groups of double cover flows. For example, we have not found $\mathbb{Z}_{2} \oplus \mathbb{Z}_{4}$ or $\mathbb{Z}_{12}$, though our work here is still very preliminary. In some cases we have $B F^{D}=B F^{+} \oplus B F^{-}$(we use a more condensed notation here and in the tables). This never seems to happen if $B F^{D}$ has infinite order. Does this say anything interesting about the flows?

TABLE 2

\begin{tabular}{|c|c|c|c|c|c|}
\hline Matrix & $P S^{+}$ & $B F^{+}$ & $P S^{-}$ & $B F^{-}$ & $B F^{D}$ \\
\hline t111111111 & $\overline{-12}$ & $\overline{\mathbb{Z}_{2}}$ & $\overline{-14}$ & $\mathbb{Z}_{4}$ & $\mathbb{Z}_{8}$ \\
\hline $1 t 1111111$ & -2 & $\mathbb{Z}_{2}$ & $\overline{0}$ & $\mathbb{Z}$ & $\mathbb{Z}$ \\
\hline$t t 1111111$ & -2 & $\mathbb{Z}_{2}$ & -2 & $\mathbb{Z}_{2}$ & $\mathbb{Z}_{2}^{2}$ \\
\hline$t 111 t 111 t t$ & -2 & $\mathbb{Z}_{2}$ & 0 & $\mathbb{Z} \oplus \mathbb{Z}_{3}$ & $\mathbb{Z} \oplus \mathbb{Z}_{3}$ \\
\hline$t t 11 t 1111$ & -2 & $\mathbb{Z}_{2}$ & -4 & $\mathbb{Z}_{4}$ & $\mathbb{Z}_{8}$ \\
\hline $1 t 1 t 11111$ & -2 & $\mathbb{Z}_{2}$ & 2 & $\mathbb{Z}_{2}$ & $\mathbb{Z}_{2}^{2}$ \\
\hline$t t t 111111$ & -2 & $\mathbb{Z}_{2}$ & 0 & $\mathbb{Z}$ & $\mathbb{Z}$ \\
\hline$t 11 t 11111$ & -2 & $\mathbb{Z}_{2}$ & -2 & $\mathbb{Z}_{2}$ & $\mathbb{Z}_{2}^{2}$ \\
\hline$t t 1 t t 1111$ & -2 & $\mathbb{Z}_{2}$ & -2 & $\mathbb{Z}_{2}$ & $\mathbb{Z}_{2}^{2}$ \\
\hline$t 111 t 1111$ & -2 & $\mathbb{Z}_{2}$ & -6 & $\mathbb{Z}_{6}$ & $\mathbb{Z}_{2} \oplus \mathbb{Z}_{6}$ \\
\hline ttttttttt & -2 & $\mathbb{Z}_{2}$ & 4 & $\mathbb{Z}_{4}$ & $\mathbb{Z}_{8}$ \\
\hline$t t t t t t 1 t t$ & -2 & $\mathbb{Z}_{2}$ & 6 & $\mathbb{Z}_{6}$ & $\mathbb{Z}_{2} \oplus \mathbb{Z}_{6}$ \\
\hline$t t 1 t t t 1 t t$ & -2 & $\mathbb{Z}_{2}$ & 0 & $\mathbb{Z} \oplus \mathbb{Z}_{3}$ & $\mathbb{Z} \oplus \mathbb{Z}_{3}$ \\
\hline $0 t 1111111$ & -3 & $\mathbb{Z}_{3}$ & -1 & 0 & $\mathbb{Z}_{3}$ \\
\hline $0111 t 1111$ & -3 & $\mathbb{Z}_{3}$ & -5 & $\mathbb{Z}_{5}$ & $\mathbb{Z}_{15}$ \\
\hline $0 t 11 t 1111$ & -3 & $\mathbb{Z}_{3}$ & -3 & $\mathbb{Z}_{3}$ & $\mathbb{Z}_{3}^{2}$ \\
\hline $01 t 1011 t 1$ & -4 & $\mathbb{Z}_{4}$ & 0 & $\mathbb{Z}$ & $\mathbb{Z} \oplus \mathbb{Z}_{2}$ \\
\hline $01 t 10111 t$ & -4 & $\mathbb{Z}_{4}$ & 0 & $\mathbb{Z}$ & $\mathbb{Z} \oplus \mathbb{Z}_{2}$ \\
\hline $011 t 011 t 1$ & -4 & $\mathbb{Z}_{4}$ & -2 & $\mathbb{Z}_{2}$ & $\mathbb{Z}_{8}$ \\
\hline $011 t 0111 t$ & -4 & $\mathbb{Z}_{4}$ & 2 & $\mathbb{Z}_{2}$ & $\mathbb{Z}_{8}$ \\
\hline $01 t t t t 110$ & -4 & $\mathbb{Z}_{4}$ & 6 & $\mathbb{Z}_{6}$ & $\mathbb{Z}_{24}$ \\
\hline $011 t 01110$ & -4 & $\mathbb{Z}_{2}^{2}$ & 0 & $\mathbb{Z} \oplus \mathbb{Z}_{2}$ & $\mathbb{Z} \oplus \mathbb{Z}_{2}^{2}$ \\
\hline
\end{tabular}


The $B F$ groups are presented in Smith Normal Form [7] and many of the calculations were done with the ismith command in Maple's linalg package. Finally, we present two twist matrices where the Bowen-Franks groups of the double covers is the only distinguishing invariant. Let

$$
A(t)=\left[\begin{array}{ccc}
3 & 1+t & 2 \\
1+t & 7 & 1+t \\
1+t & 1+t & 31
\end{array}\right] \text { and } B(t)=\left[\begin{array}{ccc}
3 & 1+t & 1+t \\
1+t & 7 & 1+t \\
1+t & 1+t & 31
\end{array}\right]
$$

Then we get

$$
\begin{aligned}
& P S^{+}=-224, B F^{+}=\mathbb{Z}_{2} \oplus \mathbb{Z}_{4} \oplus \mathbb{Z}_{28}, \\
& P S^{-}=-360, B F^{-}=\mathbb{Z}_{2} \oplus \mathbb{Z}_{6} \oplus \mathbb{Z}_{30},
\end{aligned}
$$

for both $A(t)$ and $B(t)$, but

$$
B F(D A)=\mathbb{Z}_{4} \oplus \mathbb{Z}_{24} \oplus \mathbb{Z}_{840},
$$

while

$$
B F(D B)=\mathbb{Z}_{2}^{2} \oplus \mathbb{Z}_{24} \oplus \mathbb{Z}_{840} .
$$

The author would like to thank John Franks for several helpful conversations.

\section{REFERENCES}

1. R. Bowen and J. Franks, Homology for zero-dimensional nonwandering sets, Ann. of Math. 106 (1977), 73-92. MR 56:16692

2. J. Franks, Flow equivalence of subshifts of finite type, Ergod. Th. Dynam. Sys. 4 (1984), 53-66. MR 86j:58078

3. D. Huang, Flow equivalence of reducible shifts of finite type, Ergod. Th. Dynam. Sys. 14 (1994), 695-720. MR 95k:46110

4. D. Huang, Flow equivalence of reducible shifts of finite type and Cuntz-Krieger algebras, J. Reine Angew. Math. 462 (1995), 185-217. MR 96m:46123

5. D. Huang, Flow equivalence of reducible shifts of finite type and non-simple Cuntz-Krieger algebras II: Complete classifications, Preprint.

6. Douglas Lind and Brian Marcus, An introduction to symbolic dynamics and coding, Cambridge University Press, 1995. MR 97a:58050

7. M. Newman, Integral matrices, Academic Press, 1972. MR 49:5038

8. B. Parry and D. Sullivan, A topological invariant of flows on 1-dimensional spaces, Topology 14 (1975), 297-299. MR 53:9179

9. Michael C. Sullivan, An invariant for basic sets of Smale flows, To appear in Ergod. Th. Dynam. Sys., Preprint on http://nkrs465.math.siu.edu/ mike/Preprints.

10. Michael C. Sullivan, Invariants of twist-wise flow equivalence, Preprint on http://nkrs465.math.siu.edu/ mike/Preprints. 62901

Department of Mathematics (4408), Southern Illinois University, Carbondale, IL

E-mail address: msulliva@math.siu.edu 